\title{
How Coal Miners Develop Path-Dependence and Lock in to an Unsafe Behavioral Path
}

\section{Zhengquan Xu, Qinren Cao, Ni Zhang}

School of Management, China University of Mining \& Technology, Xuzhou, China.

Email: xuzhengquan@163.com

Received November 20 ${ }^{\text {th }}, 2013$; revised December 20 ${ }^{\text {th }}, 2013$; accepted December $27^{\text {th }}, 2013$

Copyright (c) 2014 Zhengquan Xu et al. This is an open access article distributed under the Creative Commons Attribution License, which permits unrestricted use, distribution, and reproduction in any medium, provided the original work is properly cited. In accordance of the Creative Commons Attribution License all Copyrights @ 2014 are reserved for SCIRP and the owner of the intellectual property Zhengquan Xu et al. All Copyright (C 2014 are guarded by law and by SCIRP as a guardian.

\section{ABSTRACT}

Path dependence in behavioral agents can influence their actions. Creation of path dependence is a process of agents locking in their behavioral trajectory, which will increase the possibility of their certain actions occurring repeatedly. In China, most of coal mines' working place is located in deep underground, so coal miners must work in very complex environment. In such environment, once behavioral path occurred in the coal miners, the chance of incurring coal mining accidents will be heavily increased. This article provides an empirical study on how coal miners develop path-dependence and lock-in to unsafe actions. It expands upon the external and internal conditions that create path dependence in behavioral agents. Drawing on these conditions, we construct a process model of agents developing creation of path dependence and locking in their behavioral path, and then, by using the model, we elaborate why unsafe actions of coal miners in China frequently and easily occur, and why coal mine accidents reoccur in period.

\section{KEYWORDS}

\section{Unsafe Action; Path Dependence; Behavioral Agents; Accidents; Behavioral Trajectory}

\section{Introduction}

In China, unsafe actions of coal miners exhibit the characteristics of occurring frequently, easily and suddenly, and they are considered to be one of main factors which incur coal mining accidents. In practice, it is also difficult for safety supervisors to enact effective control over these unsafe actions. Although China coal mining enterprises have instituted very strict behavioral norms and invested heavily in safety equipment and techniques to prevent the unsafe actions, their effectiveness seems not very striking. Why is this happening to China coal mining? The extant literature hasn't given a reasonable answer. Analysis of accident statistics demonstrates that possibility of a certain unsafe action incurring coal mining accidents is low, but repeat of such actions will heavily increase the possibility. Heinrich notes that 330 accidents are required to produce 1 major injury, which suggests that accumulative effect is needed to induce injury accidents. Recent work on path dependence in social and management sciences has demonstrated that actions once repeated to some times will make the agents lock in a certain behavioral trajectory. The existence of path dependence in the behavioral agent raises the possibility that behavioral processes may be subject to considerable inertia. Thus path dependence can be used as the theoretical foundation for explaining why unsafe actions of China coal miners occur frequently, easily and suddenly. Most of China coal mines are located in deep underground, working places of which are very complex and change every day, therefore, someone likens the coal mine to moving factory. In such working environment, once path dependence occurred in the coal miners, the difficulty in controlling over their unsafe actions will be increased.

We construct, in this paper, a process model of how coal miners develop path-dependence and lock in to unsafe behavioral trajectory. In the model we draw on internal and external conditions of creating path depen- 
dence in behavioral agents to illustrate the process of thecoal miners being pushed in an unsafe behavioral direction.

In Section 1 of the paper, we summarize theoretical literature on path dependence, and briefly introduce the impact of path dependence on behavior choice. In Section 2, we discuss in detail the internal and external conditions of creating path dependence and their impact on behavior choice. The third section constructs a process model on how behavioral agents evolving into path dependence and locking in their behavioral trajectory, and presents a simple example. The fourth section makes conclusions.

\section{Theoretical Background of Path Dependence}

There is a lot of literature in the world of economy, management and social sciences that has illustrated importance of path dependence in exerting influence on agents' behavior choice and performance [1-3]. Literature on path dependence in the economy has emphasized ways in which economic processes can be self-reinforcing (Cowan, 1996). Self-reinforcements in the economy can lead to the existence of dynamic increasing returns and might drive the economy to an inefficient outcome [4]. Heffernan notes that inefficient technologies, institutions and social structure can be adopted and persist due to increasing returns, self-reinforcing mechanisms, and externalities [5]. Arthur has emphasized the chance that path dependence may lead to market failure [6]. Gartland argues that extant literature seldom describes impact of path dependence on an individual behavioral agent, enterprise, and organization, and mainstream economists seem almost unaware of the theoretical work in the world of social and management sciences [7-9].

The extant theoretical work on path dependence theory $[10,11]$ can help find the real reason why China coal mining accidents incurred by unsafe actions of coal miners occur repeatedly. There are three reasons for this. One is that rising coal price, special coal mining rules and complex working place facilitate coal miners to develop path dependence and lock in to unsafe behavior trajectory. The other difficulty faced by the safety supervisors is that they can't find effective measures to unlock path dependence. The third one is that coal miners in China tend to exhibit risk-taking behavior and like developing clans in their social network.

\section{Conditions of Creating Path Dependence in the Behavioral Agent}

Gartland argues that rich literature on path dependence in the world of social and management sciences does not attract much attention from mainstream economists. The key reason for this is that scholars from the world of economy almost agree on the conditions of creating path dependence in the economy, but scholars from other research world can't achieve this. The extant literature has documented the external conditions of creating path dependence in the economy or behavioral agents, but it is silent about external conditions of path dependence created by the interaction among the economy or behavioral agents. There are various social contacts and interactions among the behavioral agents because of their social properties. Thus agents' actions once generated under some conditions have a tendency to spread over the agent's social network, thereby developing externalities of the agent's social network. Integrity of social network can make the different agents' social network overlap, so that the externalities will reinforce behavior of agents in the social network and create path dependence. From above analysis, we can infer that besides internal conditions of path dependence that have been illustrated in vast literature, external conditions also exist. For the purposes of this analysis, we divide, in this article, these conditions into two types: the internal and external.

The internal conditions consist of agents' adaptability, experience accumulation, and expectation, and they form the basis of developing path dependence in the agents. Coal miners often imprecisely judge their expectation returns through the costs they spent. In practice, they often don't strictly comply with the rules and regulations, which can decrease their costs of action and increase their returns consequently. Adaptability shapes the conditions under which agents can switch from one behavioral trajectory opposed to another, so that the agents are able to adapt to the changing working environment and accumulate experience for optimizing their behavior choice. This process requires the agents to draw dynamic parallels between their expectation and outcome of behavior, make a minor adjustment to their behavioral trajectory until their behavior is locked in and path dependence is developed.

External conditions needed to create path dependence in the behavioral agents involve individual agent's social network, network externality, overlap of individual agent's social network, simplicity of behavior, and increasing returns of collective behavior. In China, most of coal mines' working places are located in deep underground at the depth of more than $600 \mathrm{~m}$. The entire underground workings are very complex and changeable, which requires the coal miners' specific division and close cooperation. To achieve this goal will lead to vast social contacts and interaction among the coal miners, and further develop individual agent's social network. One coal miner can appear in the social network of others, which cause the overlap of different coal miners' social network, and form a much larger social network 
which contains some small ones. Thus knowledge and experience accumulated by the coal miners can be promulgated over the social network and be shared, the externalities is developed, which also influences their behavior choice in the social network. For the convenience of this analysis, we can refer to two behavior choices of coal miners: the safe action and unsafe one. In comparison to safe action, the latter one can make the coal miner make less effort, save more time and money, but with higher uncertainty and risk. However, the externalities can reduce the uncertainty and risk, which will attract more coal miners to learn and imitate the unsafe actions. When unsafe actions have externalities in learning and imitating by the miners, such that the net benefit of taking an unsafe action increases with the number of the miners currently taking it, unsafe actions of the coal miners in the social network are subject to increasing returns. Chua and Lim note that clan once formed by agents who work in the complex context will increase the possibility of their collective actions' success [12]. There are two reasons for this. One is that close cooperation and coordination built during coal miners working together in the long term require them to keep frequent interaction and form groups with strong social ties. The other is that coal miners once come to agreement on the adoption of unsafe actions, they will use peer monitoring and sanction to build order and accumulate social capitals. Furthermore, the rich interaction created by social networks also foster orders sustaining and rebuilding, such that the set of unsafe actions is limited and uncertainty caused by them is reduced.

When the above internal and external conditions are satisfied, agents' behavior will be driven to reinforce, and the reinforcing effects will also be promulgated over the social networks of different agents, make more agents choose the unsafe actions rather than the safe actions that are more determinate and subject to more containments. Thus behavioral expectation, network externality, and reduction of uncertainty are three resources of developing co-reinforcing mechanism in behavioral agents in the social networks. The co-reinforcing mechanism facilitates, through the rich interaction in the social networks, the coal miners to develop path dependence and lock in to one unsafe behavioral path.

\section{Process Model on Path Dependence Developed in Coal Miners}

Creation of path dependence in the behavioral agents is a dynamic process, needs to satisfy various conditions. For these conditions, as discussed above, Figure 1 delineates their links. Drawing on the relationships among the conditions, we can construct a process model on the development of path dependence in the coal miners.

Internal conditions determine the expectation that actions adopted by behavioral agents might incur. If the returns of adopting unsafe action are more than that of safe actions, this will lead to motivation of behavioral agents adopting unsafe action. Whether the motivation can switch to active action depends on the behavioral agents' recognition on the external conditions. In China, entire underground workings of coal mining force the miners to construct rich interactions among them to recognize the various risk taking place in the process of coal

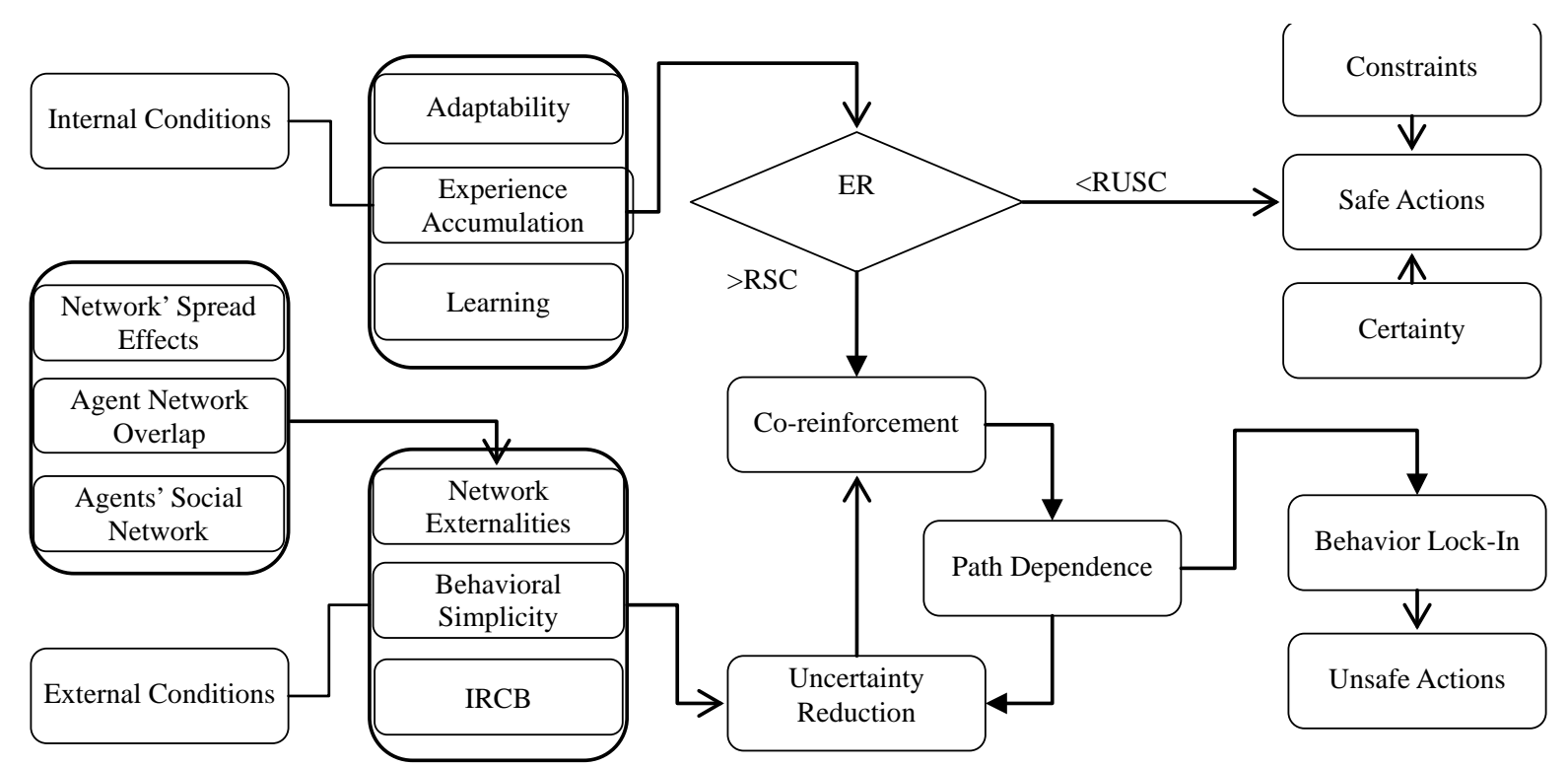

Figure 1. Process Model of Creating Path Dependence in Coal Miners. Notes: In Figure 1, IRCB denotes increasing returns of collective behavior. BE denotes expectation returns, “ $<$ RUSC” denotes that expectation returns are less than returns of unsafe actions, “>RSC” denotes that expectation returns are more than returns of safe actions. 
mining, while interaction also facilitate the accumulation of social capital and construction of social networks. Moreover, social network can create rich interaction among miners and further accumulation of social capital, foster collective actions at higher level, and improve miners' ability to recognize the working environment, so that uncertainty of taking unsafe actions is reduced. In practice, coal miners are frequently adjusting their behavioral expectation and reduce uncertainty of unsafe actions, through learning by doing, until they get approximate balance. Furthermore, the frequent adjustment of expectation interacting with the uncertainty reduction of unsafe actions through the rich feedback loops in the coal miners' social networks reinforces the unsafe actions of different agents, which also develop the co-reinforcing mechanism discussed above. While path dependence in coal miners develops in the process of their behavior being reinforced through the feedbacks in the social networks, behavior of the agents once is locked in, behavioral choice of them will depend on their experience and social capital accumulation, unless the agents suffer shock of coal mining accident or be coordinated by a new reinforcing mechanism, and be pushed to another behavioral trajectory.

Here, we present a very simple case to illustrate why coal mining accidents in China take place frequently? This case just describes the process of evolving path dependence in the coal miners. We know that the work force structure in China coal mining enterprises is very complex. Except for working relations, there exist many other relations, such as friendship, relative, fellowtownsman, and neighbor relation, among the coal miners. Although the rules and regulation in coal mining enterprises in China is very strict, yet they are not implemented well in the practice. In the last 100 years between 1878-1978 China's economy didn't make remarkable change, however, since the reform in 1978, China's economic growth has been very rapid, which lead to imbalance between supply and demand in domestic coal markets. So we can treat the reform as a contingent episode which activate the imbalance. The imbalance shapes the reinforcing mechanism, drives the coal prices, which also motivates the coal mining enterprises to increase production, even exceeding their production capacity. Some coal mining enterprises raise coal productions too rapidly, so that the rules and regulations are been implemented flexibly and some behavior of violating rules and regulations is tacitly permitted. Furthermore, entire underground workings and complex working environment in China coal mines also have negative effects on productivity, then on returns, if all rules and regulations are strictly followed. While lowering the standards of the rules and regulations will not lead the accidents to come on the heel, but rather that it can improve production and returns notably. However, the lowering requires the miner to get a proper balance between their expectations and the uncertainty caused by the lowering, and accumulate experiences and social capital to improve their cognitive ability. The above balancing is, in fact, a process of the expectation interplaying with uncertainty reduction to develop co-reinforcing mechanism. When the balance is achieved, the coal miners will have a tendency to adopt unsafe actions, even if they faced the complex working circumstance.

\section{Conclusions}

Path dependence, once developed in the coal miners, will have strong effects on their behavior choice. As path dependence creating is a process of behavioral agents obtaining behavior inertia, the agents in the face of sets of choices will be affected by their past experience to decide what to do next, and this leads the dominant actions of the agents adopting not to be optimal. Although, over a long period of time, the total returns of miners taking safe actions exceed that of unsafe actions, unsafe actions prevail in the miners in the certain span of time. This is due to three factors: externalities of social networks, uncertainty reduction of the unsafe actions and working circumstance, and improvement of cognitive ability. These factors combine to shape the positive feedbacks that reinforce unsafe actions of the miners, which make the unsafe actions more valuable as the number of coal miners taking unsafe actions increases. Thus the model, in this paper, theoretically explains the reasons why the coal miners in China have propensity to take unsafe actions.

Unsafe actions prevailing in the coal miners can evolve into accumulative effects. The possibility of one unsafe action incurring coal mining accident is low, but if it takes place repeatedly and accumulative effects are shaped, a number of the unsafe actions taking place in the coal mining process will increase the possibility that incurs the accidents. Thus unsafe actions incurring accidents exhibits a reoccurring feature. Accidents force the safety supervisors to adopt the stricter monitoring and coordinate mechanism, this combining to shock effects of the accidents drives the miners' previous behavioral trajectory to be unlocked and switch to a new behavioral path. Thus the model also accounts for the reasons why the coal mining accidents take place in period.

\section{Acknowledgements}

The authors thank the anonymous referees. They also thank the National Social Science Fund Project (Grant No. 13CGL126), MOE (Ministry of Education in China) Project of Humanities and Social Sciences (Project No. 11YJC630242) and the National Natural Science Foun- 
dation of China (Grant No. 71173217/G0312) for their support.

\section{REFERENCES}

[1] W. B. Arthur, "Increasing Returns and Path Dependence in the Economy," University of Michigan Press, Ann Arbor 1995.

[2] R. Cowen and P.Gunby, "Sprayed to Death: Path Dependence, Lock-In and Pest Control Strategies," Economic Journal, Vol. 106, No. 436, 1996, pp. 521-542. http://dx.doi.org/10.2307/2235561

[3] D. C. North, "Institutions, Institutional Change and Economic Performance,” Cambridge University Press, New York, 1990. http://dx.doi.org/10.1017/CBO9780511808678

[4] R. Cowan, "Nuclear Power Reactors: A Study in Technological Lock In,” Journal of Economic History, Vol. 50, No. 3, 1990, pp. 541-567. http://dx.doi.org/10.1017/S0022050700037153

[5] M. Heffernan, "Path Dependence, Behavioral Rules, and the Role of Entrepreneurship in Economic Change: The Case of the Automobile Industry," The Review of Austrian Economics, Vol. 16, No. 1, 2003, pp. 45-62. http://dx.doi.org/10.1023/A:1022905224020

[6] W. B. Arthur, "Competing Technologies, Increasing Returns, and Lock-In by Historical Events," Economic Journal, Vol. 99, No. 394, 1989, pp. 116-131. http://dx.doi.org/10.2307/2234208
[7] M. P.Gartland, "Interdisciplinary Views of Sub-Optimal Outcomes: Path Dependence in the Social and Management Sciences,” The Journal of Socio-Economics, Vol. 34, No. 5, 2005, pp. 686-702. http://dx.doi.org/10.1016/j.socec.2005.07.003

[8] E. Goodstein, “The Economic Roots of Environmental: Decline Property Rights or Path Dependence?” Journal of Economic Issues, Vol. 29, No. 4, 1995, pp. 1029-1043.

[9] J. Vergne and R. Durand, “The Missing Link between the Theory and Empirics of Path Dependence: Conceptual Clarification, Testability Issue, and Methodological Implications,” Journal of Management Studies, Vol. 47, No. 4, 2010, pp. 736-759. http://dx.doi.org/10.1111/j.1467-6486.2009.00913.x

[10] J. Sydow, F. Lerch and U. Staber. "Planning for Path Dependence? The Case of a Network in the BerlinBrandenburg Optics Cluster,” Economic Geography, Vol. 86, No. 2, 2010, pp. 173-195. http://dx.doi.org/10.1111/j.1944-8287.2010.01067.x

[11] R. Martin and P. Sunley, "Path dependence and regional economic evolution,” Journal of Economic Geography, Vol. 6, No. 4, 2006, pp. 395-473. http://dx.doi.org/10.1093/jeg/lbl012

[12] C. E. H. Chua, W.-K. Lim, C. Soh and S. K. Sia, "Enacting Clan Control in Complex it Projects: A Social Capital Perspective,” MIS Quarterly, Vol. 36, No. 2, 2012, pp. 577-600. 